June 2003

\title{
Effective equation of state and energy conditions in phantom/tachyon inflationary cosmology perturbed by quantum effects.
}

\author{
Shin'IChi NOJIRI ${ }^{1}$ and Sergei D. ODINTSOV ${ }^{2}$ \\ Department of Applied Physics \\ National Defense Academy, Hashirimizu Yokosuka 239-8686, JAPAN \\ A Institut d'Estudis Espacials de Catalunya, Consejo Superior de \\ Investigaciones Cientificas (IEEC/CSIC) \\ Edifici Nexus, Gran Capità 2-4, 08034, Barcelona, SPAIN and Instituciò \\ Catalana de Recerca i Estudis Avançats (ICREA)
}

\begin{abstract}
We discuss the model consisting of tachyon which may have the negative kinetic energy plus scalar phantom and plus conformal quantum matter. It is demonstrated that such a model naturally admits two deSitter phases where the early universe inflation is produced by quantum effects and the late time accelerating universe is caused by phantom/tachyon. The energy conditions bounds for such cosmology are derived. It is interesting that effective equation of state may change its sign which depends from the proper choice of the combination of phantom/tachyon and quantum effects.
\end{abstract}

PACS: 98.80.Hw,04.50.+h,11.10.Kk,11.10.Wx

\footnotetext{
${ }^{1}$ snojiri@yukawa.kyoto-u.ac.jp, nojiri@nda.ac.jp

${ }^{2}$ odintsov@ieec.fcr.es Also at Tomsk State Pedagogical University, Tomsk, RUSSIA.
} 
1. Recent astronomical data suggest the existence of the dark energy with negative pressure [1] which should provide approximately two thirds of the current universe energy density. It is expected that this dark energy is responsible for accelerating expansion of the observable universe. The ratio $w$ between the pressure $p_{d}$ and the energy density $\rho_{d}$ of the dark energy seems to be near or less than $-1,-1.62<w \equiv \frac{p_{d}}{\rho_{d}}<-0.74$ [2]. Numerous models of dark energy exist. The phantom (field with negative kinetic energy) [3] was also proposed as a candidate for dark energy as it admits negative pressure.

From another side, there is much interest now in the tachyon cosmology (see [4] and for a review, [5]) where the appearence of tachyon is basically motivated by string theory. However, standard tachyon cosmology seems to be unsatisfactory and it is expected that stringy tachyon could be important in a possible pre-inflationary open string era. Nevertheless, it is interesting that tachyon with negative kinetic energy (another type of phantom) could be introduced [6]. Such a phantom/tachyon model has naturally negative $w$. It admits the late time deSitter attractor solution and maybe considered as an interesting dark energy model[6]. However, it is clear that universe should not be so simple and different matter should be present there. In particular, it would be interesting to construct the accelerating universe with the inflationary early epoch (with possible positive or negative equation of state) and with current accelerating universe dominated by dark energy.

The purpose of the present letter is aimed in this direction. We consider a constituent model of tachyon with potential (tachyon may have the negative kinetic energy) plus standard phantom and quantum effects from the usual matter. The effective equation of state is derived and discussed in detail. It is shown that such a model admits two deSitter phases where the first phase (early universe) is produced by quantum effects and the late phase (current universe) is produced by phantom/tachyon. The restrictions from energy conditions to such cosmology are discussed.

2. The starting tachyon action is given by

$$
S_{\phi}=-\int d^{4} \sqrt{-g}\left\{V(\phi) \sqrt{1+\lambda g^{\mu \nu} \partial_{\mu} \phi \partial_{\nu} \phi}+U(\phi)\right\} .
$$

When $\lambda=1$ and $U(\phi)=0$, the above action describes the usual tachyon but when $\lambda=-1$ and $U(\phi)=0$ it corresponds to the phantom/tachyon as in [6]. 
The simplest way to account for quantum effects (at least, for CFT matter) is to include the contributions due to conformal anomaly:

$$
T=b\left(F+\frac{2}{3} \square R\right)+b^{\prime} G+b^{\prime \prime} \square R,
$$

where $F$ is the square of $4 \mathrm{~d}$ Weyl tensor, $G$ is Gauss-Bonnet invariant. In general, with $N$ scalar, $N_{1 / 2}$ spinor, $N_{1}$ vector fields, $N_{2}$ (=0 or 1 ) gravitons and $N_{\mathrm{HD}}$ higher derivative conformal scalars, $b, b^{\prime}$ and $b^{\prime \prime}$ are given by

$$
\begin{aligned}
& b=\frac{N+6 N_{1 / 2}+12 N_{1}+611 N_{2}-8 N_{\mathrm{HD}}}{120(4 \pi)^{2}} \\
& b^{\prime}=-\frac{N+11 N_{1 / 2}+62 N_{1}+1411 N_{2}-28 N_{\mathrm{HD}}}{360(4 \pi)^{2}}, \quad b^{\prime \prime}=0 .
\end{aligned}
$$

Let the metric of the 4 dimensional spacetime has the warped form:

$$
d s^{2}=-d t^{2}+L^{2} \mathrm{e}^{2 A} \sum_{i, j=1}^{3}\left(d x^{i}\right)^{2}
$$

Then the contributions due to conformal anomaly to $\rho$ and $p$ are found explicitly in $[7,8]$. The "radius" of the universe $a$ and the Hubble parameter $H$ are introduced as follows

$$
a \equiv L \mathrm{e}^{A}, \quad H=\frac{1}{a} \frac{d a}{d t}=\frac{d A}{d t} .
$$

FRW equation looks like:

$$
\begin{aligned}
H^{2} & =\frac{\kappa}{3}\left(\rho_{\phi}+\rho_{A}\right), \\
\frac{\ddot{a}}{a} & =-\frac{\kappa}{3}\left\{\frac{1}{2}\left(\rho_{\phi}+\rho_{A}\right)+\frac{3}{2}\left(p_{\phi}+p_{A}\right)\right\} .
\end{aligned}
$$

Here $\rho_{\phi}$ and $p_{\phi}$ are the energy density and the pressure of the tachyon $\phi$, respectively.

The natural assumption is that $\phi$ only depends on time $t$. Then the equation of the motion for tachyon follows from (1):

$$
0=\lambda \ddot{\phi}+\left(3 \lambda H \dot{\phi}+\frac{V^{\prime}(\phi)}{V(\phi)}\right)\left(1-\lambda \dot{\phi}^{2}\right)+\frac{U^{\prime}(\phi)}{V(\phi)}\left(1-\lambda \dot{\phi}^{2}\right)^{\frac{3}{2}} .
$$


The energy density $\rho_{\phi}$ and the pressure $p_{\phi}$ are given by

$$
\rho_{\phi}=\frac{V(\phi)}{\sqrt{1-\lambda \dot{\phi}^{2}}}+U(\phi), \quad p_{\phi}=-V(\phi) \sqrt{1-\lambda \dot{\phi}^{2}}-U(\phi) .
$$

We may further assume that the spacetime is deSitter space: $a=\mathrm{e}^{\frac{t}{L}}$. Then $[7,8]$ :

$$
\rho_{A}=-p_{A}=-\frac{6 b^{\prime}}{L^{4}} .
$$

and the FRW equations (6) and (7) are:

$$
\begin{aligned}
\frac{1}{L^{2}} & =\frac{\kappa}{3}\left(\frac{V(\phi)}{\sqrt{1-\lambda \dot{\phi}^{2}}}+U(\phi)-\frac{6 b^{\prime}}{L^{4}}\right), \\
\frac{1}{L^{2}} & =-\frac{\kappa}{3}\left(\frac{V(\phi)}{2 \sqrt{1-\lambda \dot{\phi}^{2}}}+\frac{3}{2} V(\phi) \sqrt{1-\lambda \dot{\phi}^{2}}-U(\phi)+\frac{6 b^{\prime}}{L^{4}}\right) .
\end{aligned}
$$

By combining (11) and (12), we find $\dot{\phi}=0$, that is, $\phi$ should be a constant:

$$
\phi=\phi_{0}
$$

Then Eq.(11) gives

$$
\frac{1}{L^{2}}=\frac{\kappa}{3}\left(V\left(\phi_{0}\right)+U\left(\phi_{0}\right)-\frac{6 b^{\prime}}{L^{4}}\right) \text {. }
$$

On the other hand, eq. of motion (8) gives

$$
V^{\prime}\left(\phi_{0}\right)+U^{\prime}\left(\phi_{0}\right)=0
$$

Then $V\left(\phi_{0}\right)+U\left(\phi_{0}\right)$ has an extremum at $\phi=\phi_{0}$ or $V\left(\phi_{0}\right)+U\left(\phi_{0}\right)$ is a constant. The above aruguments are valid even if $U(\phi)=0$ if $V(\phi)$ has an extremum. If we follow the proposal in [9], $V(\phi)$ has a following form:

$$
V(\phi)=V_{0}\left(1+\frac{\phi}{\phi_{0}}\right) \mathrm{e}^{-\frac{\phi}{\phi_{0}}}
$$


which is monotonically decreasing function with respect $\phi$ and has extrema at $\phi=0$, where $V(\phi)=V_{0}$, and at $\phi=+\infty, V(\phi)=0$. If, for example,

$$
U(\phi)=-V_{0} \frac{\phi}{\phi_{0}} \mathrm{e}^{-\frac{\phi}{\phi_{0}}},
$$

$V(\phi)+U(\phi)$ is always constant. Since $\dot{\phi}=0$, we find $\rho_{\phi}=-p_{\phi}=V\left(\phi_{0}\right)+$ $U\left(\phi_{0}\right)$ and therefore $w=-1$. One can solve (14) with respect to $L^{2}$ :

$$
\frac{1}{L^{2}}=-\frac{1}{4 b^{\prime} \kappa} \pm \sqrt{\frac{1}{16 b^{\prime 2} \kappa^{2}}+\frac{U_{0}}{6 b^{\prime}}} .
$$

Here

$$
U_{0} \equiv V\left(\phi_{0}\right)+U\left(\phi_{0}\right) .
$$

Since $b^{\prime}$ is usually negative, if

$$
V_{0} \leq-\frac{3}{8 b^{\prime} \kappa^{2}},
$$

the both of the solutions are real and if $V_{0}>0$, both of solutions are positive. We may consider the case that $V_{0}$ is small (more exactly $\left|b^{\prime} \kappa^{2} V_{0}\right| \ll 1$ ), then

$$
\frac{1}{L^{2}} \sim-\frac{1}{2 b^{\prime} \kappa}, \frac{\kappa U_{0}}{3} .
$$

This suggests a senario where the early universe expands by $\frac{1}{L^{2}} \sim-\frac{1}{2 b^{\prime} \kappa}$ (anomaly driven inflation) and the late universe (at present) expands by the smaller solution $\frac{1}{L^{2}} \sim \frac{\kappa U_{0}}{3}$. Eq.(21) shows that there are two regimes, one is given by almost purely quantum effects (slightly perturbed by tachyon) and another one is due to almost only tachyon perturbed by quantum effects. Then quantum induced inflation and tachyon induced one effectively decouple in such scenario.

Since now $\dot{\phi}=0$, one gets

$$
w=\frac{p_{\phi}}{\rho_{\phi}}=\frac{p_{A}}{\rho_{A}}=\frac{p_{\phi}+p_{A}}{\rho_{\phi}+\rho_{A}}=-1 .
$$

For more general case with $\dot{\phi} \neq 0$ in (9), the effective equation of state is

$$
w_{\phi} \equiv \frac{p_{\phi}}{\rho_{\phi}}=-1+\frac{\lambda \dot{\phi}^{2}\left(V(\phi)-\frac{\lambda \dot{\phi}^{2} U(\phi)}{\sqrt{1-\lambda \dot{\phi}^{2}}}\right)}{V(\phi)+U(\phi) \sqrt{1-\lambda \dot{\phi}^{2}}} .
$$


Therefore when $V(\phi)>0$ and $U(\phi) \geq 0$, if $\lambda<0$, that is, the tachyon is phantom-like, we find $w \leq-1$.

3. We may further couple the above model with the usual phantom field [10] (deSitter universe induced by phantom with quantum effects was studied in [11]). The energy density $\rho_{C}$ and pressure $p_{C}$ of the phantom field is given by $[10]$

$$
\rho_{C}=p_{C}=-\frac{C^{2}}{2},
$$

where $C$ is a constant. Denoting the energy density and pressure of the matter by $\rho_{m}$ and $p_{m}$, respectively, Eqs.(11) and (12) are modified as

$$
\begin{aligned}
\frac{1}{L^{2}}= & \frac{\kappa}{3}\left(\frac{V(\phi)}{\sqrt{1-\lambda \dot{\phi}^{2}}}+U(\phi)-\frac{6 b^{\prime}}{L^{4}}-\frac{C^{2}}{2}+\rho_{m}\right) \\
\frac{1}{L^{2}}=-\frac{\kappa}{3} & \left(\frac{V(\phi)}{2 \sqrt{1-\lambda \dot{\phi}^{2}}}+\frac{3}{2} V(\phi) \sqrt{1-\lambda \dot{\phi}^{2}}-U(\phi)+\frac{6 b^{\prime}}{L^{4}}\right. \\
& \left.-C^{2}+\frac{1}{2} \rho_{m}+\frac{3}{2} p_{m}\right) .
\end{aligned}
$$

Eqs.(25) and (26) can be solved with respect to $\rho_{m}$ and $p_{m}$ :

$$
\begin{aligned}
& \rho_{m}=-\frac{V(\phi)}{\sqrt{1-\lambda \dot{\phi}^{2}}}-U(\phi)+\frac{6 b^{\prime}}{L^{4}}+\frac{C^{2}}{2}+\frac{3}{\kappa L^{2}}, \\
& p_{m}=V(\phi) \sqrt{1-\lambda \dot{\phi}^{2}}+U(\phi)-\frac{6 b^{\prime}}{L^{4}}+\frac{C^{2}}{2}-\frac{3}{\kappa L^{2}} .
\end{aligned}
$$

Let us remind the standard energy conditions accepted in cosmology:

- Null Energy Condition (NEC): $\rho+p \geq 0$

- Weak Energy Condition (WEC): $\rho \geq 0$ and $\rho+p \geq 0$

- Strong Energy Condition (SEC): $\rho+3 p \geq 0$ and $\rho+p \geq 0$

- Dominant Energy Condition (DEC): $\rho \geq 0$ and $\rho \pm p \geq 0$

It is interesting to analyze the restrictions to current deSitter cosmology from 
the energy conditions. ${ }^{3}$ By combining (27) and (28), one gets

$$
\rho_{m}+p_{m}=C^{2}-\frac{\lambda \dot{\phi}^{2} V(\phi)}{\sqrt{1-\lambda \dot{\phi}^{2}}} .
$$

Then NEC can be satisfied if $V(\phi)>0, \lambda<0$, which is the case that the tachyon is phantom-like. The expression of $\rho_{m}(27)$ can be rewritten as

$$
\begin{aligned}
\rho_{m}= & \frac{\beta(\phi)}{2 L^{4}}\left(L^{4}-\frac{6}{\kappa \beta(\phi)} L^{2}+\frac{12 b^{\prime}}{\beta(\phi)}\right) \\
= & \frac{\beta(\phi)}{2 L^{4}}\left(L^{2}-\frac{3}{\kappa \beta(\phi)}+\sqrt{\left(\frac{3}{\kappa \beta(\phi)}\right)^{2}-\frac{12 b^{\prime}}{\beta(\phi)}}\right) \\
& \times\left(L^{2}-\frac{3}{\kappa \beta(\phi)}-\sqrt{\left.\left(\frac{3}{\kappa \beta(\phi)}\right)^{2}-\frac{12 b^{\prime}}{\beta(\phi)}\right)}\right. \\
\beta(\phi) \equiv & \frac{C^{2}}{2}-\frac{V(\phi)}{\sqrt{1-\lambda \dot{\phi}^{2}}}-U(\phi)
\end{aligned}
$$

If $\beta(\phi)<0, \rho_{m}<0$, then WEC or DEC is not satisfied. If $\beta(\phi)>0$, since $L^{2}-\frac{3}{\kappa \beta(\phi)}+\sqrt{\left(\frac{3}{\kappa \beta(\phi)}\right)^{2}-\frac{12 b^{\prime}}{\beta(\phi)}}>0$, WEC or DEC gives a non-trivial constraint on $L^{2}$ :

$$
L^{2}>\frac{3}{\kappa \beta(\phi)}+\sqrt{\left(\frac{3}{\kappa \beta(\phi)}\right)^{2}-\frac{12 b^{\prime}}{\beta(\phi)}} .
$$

We also have

$$
\begin{aligned}
\rho_{m}+3 p_{m}= & \frac{\gamma(\phi)}{L^{4}}\left(L^{4}-\frac{6}{\kappa \gamma(\phi)} L^{2}-\frac{12 b^{\prime}}{\gamma(\phi)}\right) \\
= & \frac{\gamma(\phi)}{L^{4}}\left(L^{2}-\frac{3}{\kappa \gamma(\phi)}+\sqrt{\left(\frac{3}{\kappa \gamma(\phi)}\right)^{2}+\frac{12 b^{\prime}}{\gamma(\phi)}}\right) \\
& \times\left(L^{2}-\frac{3}{\kappa \gamma(\phi)}-\sqrt{\left.\left(\frac{3}{\kappa \gamma(\phi)}\right)^{2}+\frac{12 b^{\prime}}{\gamma(\phi)}\right)},\right.
\end{aligned}
$$

\footnotetext{
${ }^{3}$ Generally speaking, the energy conditions bounds are not dictated by some deep physical principle.
} 


$$
\gamma(\phi) \equiv 2 C^{2}-\frac{V(\phi)}{\sqrt{1-\lambda \dot{\phi}^{2}}}+3 V(\phi) \sqrt{1-\lambda \dot{\phi}^{2}}+2 U(\phi) .
$$

When $V(\phi), U(\phi)>0$ and $\lambda<0$, we find $\gamma(\phi)>0$. In this case, if the quantity inside the squre root is negative:

$$
\left(\frac{3}{\kappa \gamma(\phi)}\right)^{2}+\frac{12 b^{\prime}}{\gamma(\phi)}<0
$$

we obtain $\rho_{m}+3 p_{m}>0$ and SEC is satisfied. On the other hand, if

$$
\left(\frac{3}{\kappa \gamma(\phi)}\right)^{2}+\frac{12 b^{\prime}}{\gamma(\phi)}>0
$$

SEC gives a non-trivial constraint on $L^{2}$

$$
L^{2}<\frac{3}{\kappa \gamma(\phi)}-\sqrt{\left(\frac{3}{\kappa \gamma(\phi)}\right)^{2}+\frac{12 b^{\prime}}{\gamma(\phi)}} \text { or } L^{2}>\frac{3}{\kappa \gamma(\phi)}+\sqrt{\left(\frac{3}{\kappa \gamma(\phi)}\right)^{2}+\frac{12 b^{\prime}}{\gamma(\phi)}} .
$$

One also has

$$
\begin{aligned}
\rho_{m}-p_{m}= & -\frac{\eta(\phi)}{L^{4}}\left(L^{4}-\frac{6}{\kappa \eta(\phi)} L^{2}-\frac{12 b^{\prime}}{\eta(\phi)}\right) \\
= & -\frac{\eta(\phi)}{L^{4}}\left(L^{2}-\frac{3}{\kappa \eta(\phi)}+\sqrt{\left(\frac{3}{\kappa \eta(\phi)}\right)^{2}+\frac{12 b^{\prime}}{\eta(\phi)}}\right) \\
& \times\left(L^{2}-\frac{3}{\kappa \eta(\phi)}-\sqrt{\left.\left(\frac{3}{\kappa \eta(\phi)}\right)^{2}+\frac{12 b^{\prime}}{\eta(\phi)}\right)},\right. \\
\eta(\phi) \equiv & \frac{V(\phi)}{\sqrt{1-\lambda \dot{\phi}^{2}}}+V(\phi) \sqrt{1-\lambda \dot{\phi}^{2}}+2 U(\phi) .
\end{aligned}
$$

Now if $V(\phi), U(\phi)>0$, then $\eta(\phi)>0$ and DEC gives a constraint:

$$
\frac{3}{\kappa \eta(\phi)}-\sqrt{\left(\frac{3}{\kappa \eta(\phi)}\right)^{2}+\frac{12 b^{\prime}}{\eta(\phi)}}<L^{2}<\frac{3}{\kappa \eta(\phi)}+\sqrt{\left(\frac{3}{\kappa \eta(\phi)}\right)^{2}+\frac{12 b^{\prime}}{\eta(\phi)}} .
$$


As a special case, we consider the dust as a matter, where $p_{m}=0$. Then Eq.(28) gives

$$
\begin{aligned}
L^{2} & =\frac{3}{\kappa \xi(\phi)} \pm \sqrt{\left(\frac{3}{\kappa \xi(\phi)}\right)^{2}+\frac{12 b^{\prime}}{\xi(\phi)}}, \\
\xi(\phi) & \equiv \frac{C^{2}}{2}+V(\phi) \sqrt{1-\lambda \dot{\phi}^{2}}+U(\phi)
\end{aligned}
$$

Note that $\xi(\phi)$ is positive if $V(\phi), U(\phi)>0$. On the other hand, say, from (33), we obtain

$$
\rho_{m}=C^{2}-\frac{\lambda \dot{\phi}^{2} V(\phi)}{\sqrt{1-\lambda \dot{\phi}^{2}}} .
$$

Then if $V(\phi)>0, \lambda<0$, which is the case that the tachyon is phantom-like, all the energy conditions are satisfied.

When

$$
\left|\kappa^{2} b^{\prime} \xi(\phi)\right| \ll 1
$$

two solutions (42) are:

$$
L^{2}=\frac{6}{\kappa \xi(\phi)}, \quad-2 b^{\prime} \kappa^{2}
$$

The first one corresponds to the classical tachyon solution. On the other hand, the second solution is induced by the quantum corrections and is independent of the tachyon $\phi$ or phantom $C$. Then as in (21), there are two decoupled deSitter phases. One is induced by quantum effects and another one is due to the classical tachyon and phantom.

On the other hand, in (42), we may consider the case that the quantum contribution has almost same magnitude with that from the phantom and tachyon:

$$
\left|\kappa^{2} b^{\prime} \xi(\phi)\right| \sim 1
$$

Then two solutions (42) are of the same order with each other. Especially when

$$
\kappa^{2} b^{\prime} \xi(\phi)=-1,
$$

the solutions become degenerate and there is only one solution. The magnitude of $L^{2}$ in the degenerate case is half of the classical case that $b^{\prime}=0$. If

$$
\kappa^{2} b^{\prime} \xi(\phi)<-1
$$


the solutions (42) become imaginary. This is indication to (non-physical) oscillating metric.

Let us investigate the effective equation of state, i.e. $w$ including all the dark-side: tachyon, phantom and quantum corrections. By combining (9), (10), and (24), one finds

$$
\begin{aligned}
w & \equiv \frac{p_{\phi}+p_{A}+p_{C}}{\rho_{\phi}+\rho_{A}+\rho_{C}} \\
& =\frac{-V(\phi) \sqrt{1-\lambda \dot{\phi}^{2}}-U(\phi)+\frac{6 b^{\prime}}{L^{4}}-\frac{C^{2}}{2}}{\frac{V(\phi)}{\sqrt{1-\lambda \dot{\phi}^{2}}}+U(\phi)-\frac{6 b^{\prime}}{L^{4}}-\frac{C^{2}}{2}} \\
& =1-\frac{V(\phi)\left(\sqrt{1-\lambda \dot{\phi}^{2}}+\frac{1}{\sqrt{1-\lambda \dot{\phi}^{2}}}\right)+U(\phi)-\frac{6 b^{\prime}}{L^{4}}}{\frac{V(\phi)}{\sqrt{1-\lambda \dot{\phi}^{2}}}+U(\phi)-\frac{6 b^{\prime}}{L^{4}}-\frac{C^{2}}{2}} .
\end{aligned}
$$

In the limit of $C \rightarrow \infty, w \rightarrow 1$. On the other hand, when $C \rightarrow 0$, we find

$$
\begin{aligned}
w & \rightarrow \frac{-V(\phi) \sqrt{1-\lambda \dot{\phi}^{2}}-U(\phi)+\frac{6 b^{\prime}}{L^{4}}}{\frac{V(\phi)}{\sqrt{1-\lambda \dot{\phi}^{2}}}+U(\phi)-\frac{6 b^{\prime}}{L^{4}}} \\
& =-1+\frac{\lambda \dot{\phi}^{2} V(\phi)}{\sqrt{1-\lambda \dot{\phi}^{2}}\left(\frac{V(\phi)}{\sqrt{1-\lambda \dot{\phi}^{2}}}+U(\phi)-\frac{6 b^{\prime}}{L^{4}}\right)},
\end{aligned}
$$

which is less than -1 if $\lambda<0, U, V>0$, and $b^{\prime}<0$. The obtained expression (50) is a monotonically decreasing function of $C$ in the region $0<\frac{C^{2}}{2}<$ $\frac{V(\phi)}{\sqrt{1-\lambda \dot{\phi}^{2}}}+U(\phi)-\frac{6 b^{\prime}}{L^{4}}$ and $\frac{V(\phi)}{\sqrt{1-\lambda \dot{\phi}^{2}}}+U(\phi)-\frac{6 b^{\prime}}{L^{4}}<\frac{C^{2}}{2}<\infty$. There is a pole singularity at $\frac{C^{2}}{2}=\frac{V(\phi)}{\sqrt{1-\lambda \dot{\phi}^{2}}}+U(\phi)-\frac{6 b^{\prime}}{L^{4}}$. In the limit $\frac{C^{2}}{2} \rightarrow \frac{V(\phi)}{\sqrt{1-\lambda \dot{\phi}^{2}}}+U(\phi)-$ $\frac{6 b^{\prime}}{L^{4}}-0$, we obtain $w \rightarrow-\infty$. Then in the region $\frac{C^{2}}{2} \sim \frac{V(\phi)}{\sqrt{1-\lambda \dot{\phi}^{2}}}+U(\phi)-\frac{6 b^{\prime}}{L^{4}}$ but $\frac{C^{2}}{2}<\frac{V(\phi)}{\sqrt{1-\lambda \dot{\phi}^{2}}}+U(\phi)-\frac{6 b^{\prime}}{L^{4}}$, one may get large negative $w$. When $\frac{C^{2}}{2} \sim$ $\frac{V(\phi)}{\sqrt{1-\lambda \dot{\phi}^{2}}}+U(\phi)-\frac{6 b^{\prime}}{L^{4}}$, from Eqs.(27) and (28), we find

$$
\begin{aligned}
& \rho_{m} \sim \frac{3}{\kappa L^{2}} \\
& p_{m} \sim V(\phi)\left(\sqrt{1-\lambda \dot{\phi}^{2}}+\frac{1}{\sqrt{1-\lambda \dot{\phi}^{2}}}\right)+2 U(\phi)-\frac{12 b^{\prime}}{L^{4}}-\frac{3}{\kappa L^{2}} .
\end{aligned}
$$


Then NEC (29) and WEC (30) are satisfied. SEC (31) is satisfied if

$$
3 V(\phi)\left(\sqrt{1-\lambda \dot{\phi}^{2}}+\frac{1}{\sqrt{1-\lambda \dot{\phi}^{2}}}\right)+6 U(\phi)-\frac{36 b^{\prime}}{L^{4}} \geq \frac{6}{\kappa L^{2}} .
$$

and DEC (32) is satisfied if

$$
V(\phi)\left(\sqrt{1-\lambda \dot{\phi}^{2}}+\frac{1}{\sqrt{1-\lambda \dot{\phi}^{2}}}\right)+2 U(\phi)-\frac{12 b^{\prime}}{L^{4}} \leq \frac{6}{\kappa L^{2}} .
$$

Eqs.(53) and (54) show that SEC and DEC do not conflict with each other. Although the value of $L^{2}$ depends on the contribution of the matter energy density, Eqs.(53) and (54) give non-trivial constraints on $L^{2}$. If

$$
\zeta(\phi) \equiv V(\phi)\left(\sqrt{1-\lambda \dot{\phi}^{2}}+\frac{1}{\sqrt{1-\lambda \dot{\phi}^{2}}}\right)+2 U(\phi)>0,
$$

Eqs.(53) and (54) can be rewritten as

$$
L^{2} \leq \frac{1}{\kappa^{2} \zeta(\phi)}-\sqrt{\frac{1}{\kappa^{4} \zeta(\phi)^{2}}+\frac{12 b^{\prime}}{\zeta(\phi)}} \quad \text { or } L^{2} \geq \frac{1}{\kappa^{2} \zeta(\phi)}+\sqrt{\frac{1}{\kappa^{4} \zeta(\phi)^{2}}+\frac{12 b^{\prime}}{\zeta(\phi)}} .
$$

On the other hand, Eq.(54) can be rewritten as

$$
\frac{3}{\kappa^{2} \zeta(\phi)}-\sqrt{\frac{9}{\kappa^{4} \zeta(\phi)^{2}}+\frac{12 b^{\prime}}{\zeta(\phi)}} \leq L^{2} \leq \frac{3}{\kappa^{2} \zeta(\phi)}+\sqrt{\frac{9}{\kappa^{4} \zeta(\phi)^{2}}+\frac{12 b^{\prime}}{\zeta(\phi)}} .
$$

Then all the energy conditions can be satisfied if

$$
\begin{aligned}
\frac{3}{\kappa^{2} \zeta(\phi)}-\sqrt{\frac{9}{\kappa^{4} \zeta(\phi)^{2}}+\frac{12 b^{\prime}}{\zeta(\phi)}} \leq L^{2} \leq \frac{1}{\kappa^{2} \zeta(\phi)}-\sqrt{\frac{1}{\kappa^{4} \zeta(\phi)^{2}}+\frac{12 b^{\prime}}{\zeta(\phi)}} \\
\text { or } \quad \frac{1}{\kappa^{2} \zeta(\phi)}+\sqrt{\frac{1}{\kappa^{4} \zeta(\phi)^{2}}+\frac{12 b^{\prime}}{\zeta(\phi)}} \leq L^{2} \leq \frac{3}{\kappa^{2} \zeta(\phi)}+\sqrt{\frac{9}{\kappa^{4} \zeta(\phi)^{2}}+\frac{12 b^{\prime}}{\zeta(\phi)}}(58)
\end{aligned}
$$

We also note that in the limit $\frac{C^{2}}{2} \rightarrow \frac{V(\phi)}{\sqrt{1-\lambda \dot{\phi}^{2}}}+U(\phi)-\frac{6 b^{\prime}}{L^{4}}+0$ (instead of $\left.\frac{C^{2}}{2} \rightarrow \frac{V(\phi)}{\sqrt{1-\lambda \dot{\phi}^{2}}}+U(\phi)-\frac{6 b^{\prime}}{L^{4}}-0\right)$, we obtain $w \rightarrow+\infty$. Then when the 
contribution of the phantom has almost the same magnitude with those from the tachyon and the quantum effects but that of the phantom is a little bit bigger, $w$ becomes positive. Note that the sign of the contribution to the pressure from the phantom is opposite to those from the tachyon and the quantum corrections. Then the sign change of $w$ occurs when the sign of the pressure is changed. We should also note that $w$ corresponding to only tachyon is given by (23), which is less than or equal to -1 when $V(\phi)>$ $0, U(\phi) \geq 0, \lambda<0$. On the other hand $w$ corresponding to quantum corrections is -1 as in (22). Eq.(24) tells $w$ corresponding to phantom is unity. The reason why $w$ can be positive when $\frac{C^{2}}{2}>\frac{V(\phi)}{\sqrt{1-\lambda \dot{\phi}^{2}}}+U(\phi)-\frac{6 b^{\prime}}{L^{4}}$ but $\frac{C^{2}}{2} \sim \frac{V(\phi)}{\sqrt{1-\lambda \dot{\phi}^{2}}}+U(\phi)-\frac{6 b^{\prime}}{L^{4}}+0$ is surely due to the existence of the phantom. Moreover, $w$ can become large due to the proper choice of combination for phantom, tachyon and the quantum effects.

When $\dot{\phi}$ is small, the role of quantum effects is just to shift the contribution from the tachyon:

$V(\phi) \sqrt{1-\lambda \dot{\phi}^{2}}+U(\phi) \sim \frac{V(\phi)}{\sqrt{1-\lambda \dot{\phi}^{2}}}+U(\phi) \sim V(\phi)+U(\phi) \rightarrow V(\phi)+U(\phi)-\frac{6 b^{\prime}}{L^{4}}$.

The quantum effects become dominant for small $L$ even if $C$ is large (compare with [11]). In the limit where $L$ is small, $w(50)$ goes to -1 .

The main lesson drawn from this study is that constituent dark energy model may provide several deSitter-like phases of accelerated expansion where effective equation of state may change its sign when fine-tuning the model. The role of quantum effects is to drive the early universe to inflationary epoch. In some situations, bounds provided by the standard energy conditions maybe satisfied.

Acknowledgments. The research is supported in part by the Ministry of Education, Science, Sports and Culture of Japan under the grant n.13135208 (S.N.), DGI/SGPI (Spain) project BFM2000-0810 (S.D.O.), RFBR grant 0301-00105 (S.D.O.) and LRSS grant 1252.2003 .2 (S.D.O.). 


\section{References}

[1] S. Perlmutter et al., Nature, 391 (1998) 51; S. Perlmutter et al., Astrophys.J. 517 (1999) 565; A. Riess et al., Astron.J. 116 (1998), 1009.

[2] A. Melchiorri, L. Mersini, C.J. Odmann and M. Trodden, astro$\mathrm{ph} / 0211522$.

[3] R.R. Caldwell, Phys.Lett. B545(2002) 23; L. Parker and A. Raval, Phys.Rev. D60 (1999) 063512; T. Chiba, T. Okabe and M. Yamaguchi, Phys.Rev. D62 (2000) 023511; B. Boisseau, G. Esposito-Farese, D. Polarski and A.A. Starobinsky, Phys.Rev.Lett. 85 (2000) 2236; A.E. Schulz and M. White, Phys.Rev. D64 (2001) 043514; V. Faraoni, Int.J.Mod.Phys. D64 (2002) 043514; I. Maor, R. Brustein, J. Mcmahon and P.J. Steinhardt, Phys.Rev. D65 (2002) 123003; V.K. Onemli and R.P. Woodard, Class.Quant.Grav. 19 (2002) 4607; D.F. Torres, Phys.Rev. D66 (2002) 043522; S.M. Carroll, M. Hoffman and M. Trodden, astro-ph/0301273; P.H. Frampton, hep-th/0302007; S. Nojiri and S.D. Odintsov, hep-th/0304131, Phys.Lett. B, to appear; A. Feinstein and S. Jhingan, hep-th/0304069; L.P. Chimento and A. Feinstein, astroph/0305007; P. Singh, M. Sami and N. Dadhich, hep-th/0305110.

[4] A. Sen, JHEP 0204 (2002) 048, JHEP 0207 (2002) 065, hepth/0303057; G.W. Gibbons, Phys.Lett. B537 (2002) 1; M. Fairbairn and M.H.G. Tytgat, Phys.Lett. B546 (2002) 1; G.W. Gibbons, K. Hori and P. Yi, Nucl.Phys. B596 (2001) 136; J.G. Hao and X.Z. Li, Phys.Rev. D66 (2002) 087301; A. Feinstein,Phys.Rev. D66 (2002) 063511; S. Mukohyama, Phys.Rev. D66 (2002) 024009; M. Sami, P. Chingangbam, T. Qureshi, Phys.Rev. D66 (2002) 043530; M. Sami and T. Padmanabhan, Phys.Rev. D67 (2003) 083509; G. Shiu and I. Wasserman, Phys.Lett. B541 (2002) 6; H.B. Benaoum, hep-th/0205140; A. Ishida and S. Uehara, hep-th/0206102; T. Chiba, astro-ph/0206298; T. Mehen and B. Wecht, hep-th/0206212; N. Moeller and B. Zwiebach, JHEP 0210 (2002) 034; Y.-S. Piao, R.-G. Cai, X.-m. Zhang, Y.-Z. Zhang, Phys.Rev. D66 (2002) 121301; Y.-S. Piao, Q.-G. Huang, X.-m. Zhang, Y.-Z. Zhang, hep-ph/0212219; J.M. Cline, H. Firouzjahi and P. Martineau, hep-th/0207156; T. Okuda and S. Sugimoto, hep-th/0208196; G. Gibbons , K. Hashimoto and P. Yi, hep-th/0209034; M.R. Garousi, 
hep-th/0209068; B. Chen, M. Li and F. Lin, hep-th/0209222; J. Luson, hep-th/0209255; C. Kim, H.B. Kim, Y. Kim and O. K. Kwon, hep-th/0301142; X.Z. Li, D.J. Liu and J.G. Hao, hep-th/0207146; G. Felder, L. Kofman and A. Starobinsky, JHEP 0209 (2002) 026; G.A. Diamandis, B.C. Georgalas, N.E. Mavromatos, E. Papantonopoulos and I. Pappa, hep-th/0107124; M.C. Bento, O. Bertolami and A.A. Sen, Phys.Rev. D67 (2003) 023504; C. Kim, Y. Kim, O.K. Kwon and C. Oh Lee, hep-th/0305092; H. Lee and W.S. l'Yi, hep-th/0210221; J.S. Bagla, H.K. Jassal and T. Padmanabhan, astro-ph/0212198; F. Leblond and A.W. Peet, hep-th/0303035; T. Matsuda, hep-ph/0302035; A. Das and A. DeBenedictis, gr-qc/0304017; A. Majumdar and A. Davis, hepth/0304226.

[5] G. Gibbons, hep-th/0301117.

[6] J.-g. Hao and X.-z. Li, hep-th/0305207; hep-th/0306033.

[7] S. Nojiri and S.D. Odintsov, Int.J.Mod.Phys. A16 (2001) 3273, hepth/0011115.

[8] S. Nojiri, S.D. Odintsov and S. Ogushi, Int.J.Mod.Phys. A17 (2002) 4809, hep-th/0205187.

[9] D. Kutasov, M. Marino and G.W. Moore, JHEP 045 (2000) 0010.

[10] G.W. Gibbons, hep-th/0302199.

[11] S. Nojiri and S.D. Odintsov, Phys.Lett. B562 (2003) 147, hepth/0303117. 